\title{
Modeling cortical maps with Topographica
}

\author{
James A. Bednar ${ }^{\mathrm{a}}$, Yoonsuck Choe ${ }^{\mathrm{b}}$, Judah De Paula ${ }^{\mathrm{a}}$, Risto Miikkulainen ${ }^{\mathrm{a}}$, \\ Jefferson Provost ${ }^{\mathrm{a}}$, and Tal Tversky ${ }^{\mathrm{a}}$ \\ ${ }^{a}$ Department of Computer Sciences, The University of Texas at Austin, Austin, TX 78712 \\ \{jbednar, tal, jp, judah, risto\} ecs.utexas.edu \\ ${ }^{\mathrm{b}}$ Department of Computer Science, Texas A\&M University, TAMU 3112, College Station, TX 77843 \\ choeetamu.edu
}

\begin{abstract}
The biological function of cortical neurons can often be understood only in the context of large, highly interconnected networks. These networks typically form two-dimensional topographic maps, such as the retinotopic maps in the visual system. Computational simulations of these areas have led to valuable insights about how cortical topography develops and functions, but further progress is difficult because appropriate simulation tools are not available. This paper introduces the freely available Topographica map-level simulator, currently under development at the University of Texas at Austin. Topographica is designed to make large-scale, detailed models practical. The goal is to allow neuroscientists and computational scientists to understand how topographic maps and their connections organize and operate. This understanding will be crucial for integrating experimental observations into a comprehensive theory of cortical function.
\end{abstract}

Key words: simulation tools, cortical modeling, topographic maps, self-organization, development

\section{Introduction}

Much of the cortex of mammals can be partitioned into topographic maps [8, 13]. These maps contain systematic two-dimensional representations of features relevant to sensory and motor processing, such as retinal position, sound frequency, line orientation, and motion direction [5, 9, 14]. Understanding the development and function of topographic maps is crucial for understanding brain function, and will require integrating large-scale experimental imaging results with single-unit studies of individual neurons and their connections.

Computational simulations have proven to be a powerful tool in this endeavor. In a simulation, it is possible to explore how topographic maps can emerge from the behavior of single neurons, both during development and during perceptual and motor processing in the adult. (For a review of this class of models, see [12].) However, the models to date have been limited in size and scope because existing simulation tools do not provide specific support for biologically realistic, densely interconnected topographic maps. Existing biological neural simulators, such as NEURON [7] and GENESIS [6], primarily focus on detailed studies of individual neurons 


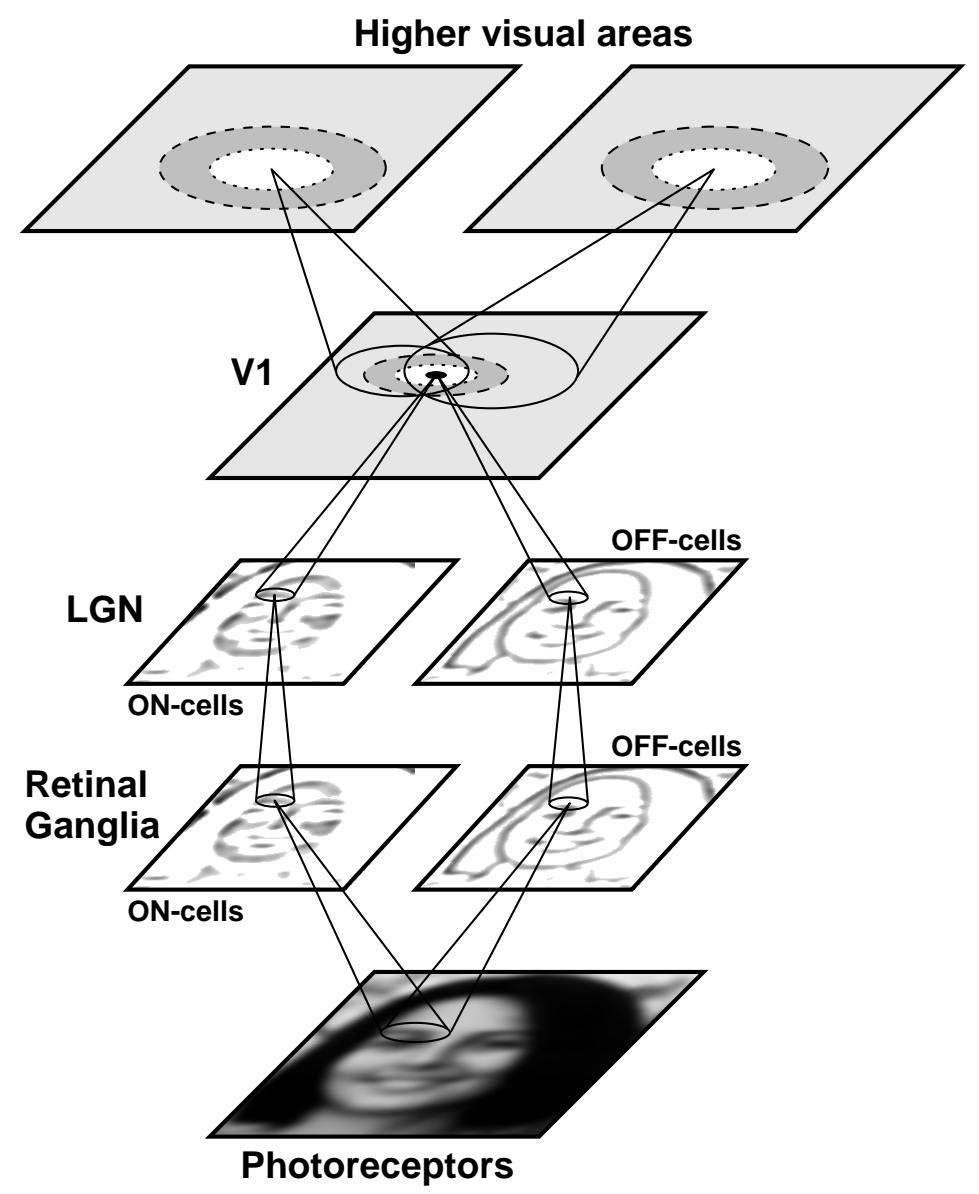

Fig. 1. Topographica models. This figure shows a sample Topographica model of the early visual system [3, 4]. In Topographica, models are composed of interconnected sheets of neurons. Each visual area in this model is represented by one or more sheets. For instance, the eye is represented by three sheets: a sheet representing an array of photoreceptors, plus two sheets representing retinal ganglion cells. Each of the sheets can be coarse or detailed, plastic or fixed, as needed for a particular study. Sheets are connected to other sheets, and units within each sheet can be connected using lateral connections. For one cell in each sheet in the figure, sample connections are shown, including lateral connections in V1 and higher areas. Similar models can be used for topographic maps in somatosensory, auditory, and motor cortex.

or very small networks of them. Tools for simulating large populations of abstract units, such as PDP++ [10] and Matlab (www.mathworks.com), focus on cognitive science and engineering applications, rather than models of cortical areas. As a result, current simulators also lack specific support for measuring topographic map structure or generating input patterns at the topographic map level.

This paper introduces the Topographica map-level simulator, which is designed to make it practical to simulate large-scale, detailed models of topographic maps. Topographica is designed to complement the existing low-level and abstract simulators, focusing on biologically realistic networks of tens of thousands of neurons, forming topographic maps containing millions or tens of millions of connections. Topographica is being developed at the University of Texas at Austin as part of the Human Brain Project of the National Institutes of Mental Health. Topographica is an open source project, and binaries and source code will be freely available through the internet at topographica.org. In the sections below, we describe the models and modeling approaches supported by Topographica, how the simulator is implemented, and how it can be used to advance the field of computational neuroscience.

\section{Scope and design}

Figure 1 illustrates the types of models supported by Topographica. The models focus on topographic maps in any two-dimensional cortical or subcortical region, such as visual, auditory, somatosensory, proprioceptive, and motor maps. Typically, models will include multiple re- 
gions, such as an auditory or visual processing pathway, and simulate a large enough area to allow the organization and function of each map to be studied. The external environment must also be simulated, including playback of visual images, audio recordings, and test patterns. Current models typically include only a primary sensory area with a simplified version of an input pathway, but larger scale models will be crucial for understanding phenomena such as object perception, scene segmentation, speech processing, and motor control. Topographica is intended to support the development of such models.

To make it practical to model topographic maps at this large scale, the fundamental unit in the simulator is a two-dimensional sheet of neurons, rather than a neuron or a part of a neuron. Conceptually, a sheet is a continuous, two-dimensional area (as in [1, 11]), which is typically approximated by a finite array of neurons. This approach is crucial to the simulator design, because it allows user parameters, model specifications, and interfaces to be independent of the details of how each sheet is implemented.

As a result, the user can easily trade off between simulation detail and computational requirements, depending on the specific phenomena under study in a given simulator run. (See [2] for more details on model scaling.) If enough computational power and experimental measurements are available, models can be simulated at full scale, with as many neurons and connections as in the animal system being studied. More typically, a less-dense approximation will be used, requiring only ordinary PC workstations. Because the same model specifications and parameters can be used in each case, switching between levels of analysis does not require extensive parameter tuning or debugging as would be required in neuron-level or engineering-oriented simulators.

For most simulations, the individual neuron models within sheets can be implemented at a high level, consisting of single-compartment firing-rate or integrate-and-fire units. More detailed neuron models can also be used, when required for experimental validation or to simulate specific phenomena. These models will be implemented using interfaces to existing low-level simulators such as NEURON and GENESIS.

\section{Implementation}

Topographica consists of a graphical user interface (GUI), scripting language, and libraries of models, analysis routines, and visualizations. The model library consists of predefined types of sheets, connections, neuron models, and learning rules, and can be extended with user-defined components. These building blocks are combined into a model using the GUI and (when necessary) the script language.

The analysis and visualization libraries include statistical tests and plotting capabilities geared towards large, two-dimensional areas. They also focus on data displays that can be compared with experimental results, such as optical imaging recordings, for validating models and for generating predictions. Figure 2 shows examples of the visualization types currently supported. This figure is a screenshot from a prototype version of Topographica, available for download at topographica.org.

To allow large models to be executed quickly, the numerically intensive portions of the simulator 


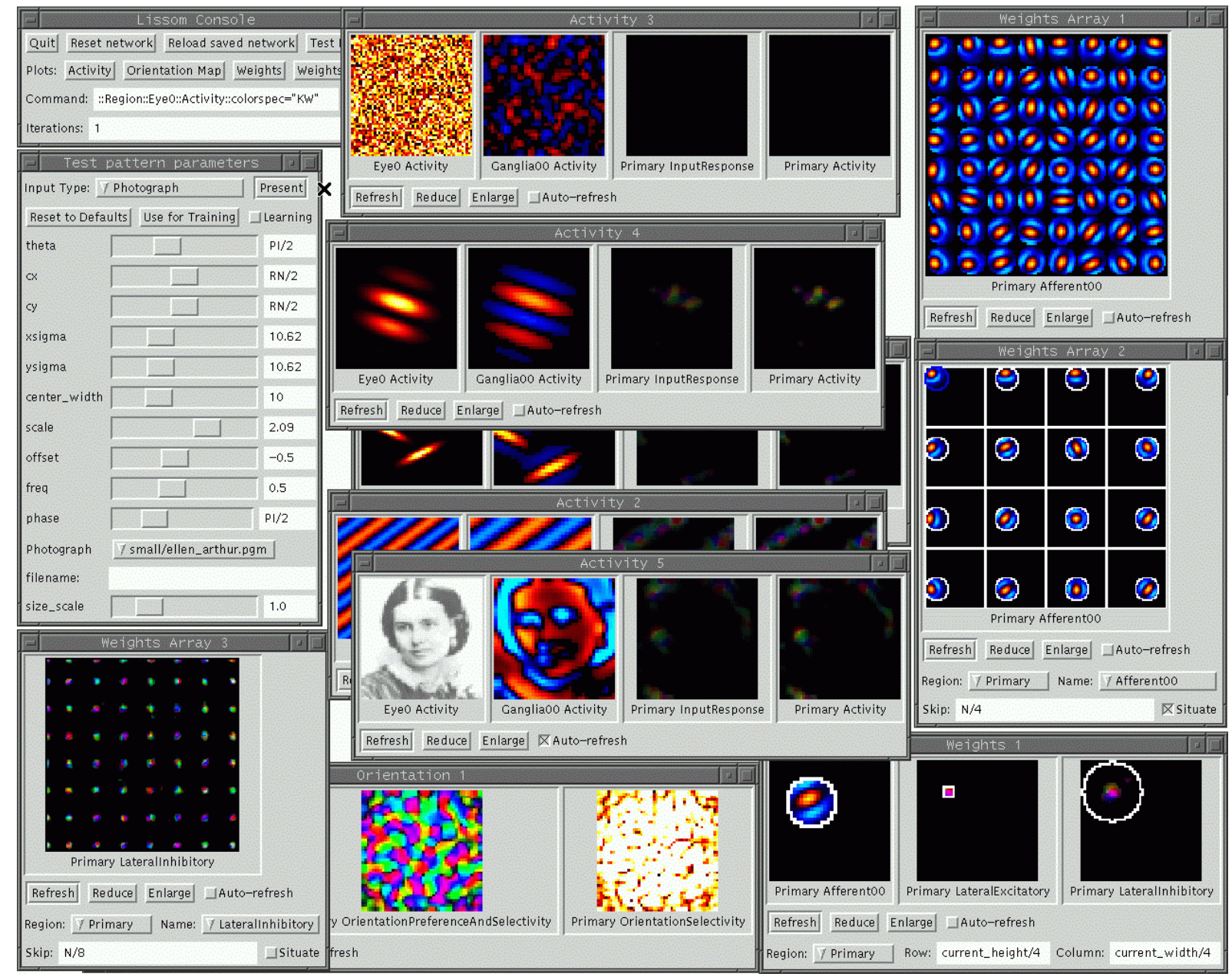

Fig. 2. Software screenshot. This image shows a sample session of a prototype version of Topographica that is available freely at topographica.org. Here the user is studying the behavior of an orientation map in the primary visual cortex (V1), using a model similar to the one depicted in figure 1 . The window at the bottom labeled "Orientation" shows the self-organized orientation map and the orientation selectivity in V1. The windows labeled "Activity" show a sample visual image on the left, along with the responses of the retinal ganglia and V1 (labeled "Primary"). The input patterns were generated using the "Test pattern parameters" dialog at the left. The window labeled "Weights" shows the strengths of the connections to one neuron in V1. This neuron has afferent receptive fields in the ganglia and lateral receptive fields within $\mathrm{V} 1$. The afferent weights for an $8 \times 8$ and $4 \times 4$ sampling of the V1 neurons are shown in the "Weights Array" windows on the right; most neurons are selective for Gabor-like patches of oriented lines. The lateral connections for an $8 \times 8$ sampling of neurons are shown in the "Weights Array" window at the lower left; neurons tend to connect to their immediate neighbors and to distant neurons of the same orientation. This type of large-scale analysis is difficult with existing simulators, but Topographica is well suited for it. See topographica. org for a color version of this figure.

are implemented in $\mathrm{C}++$. Equally important, however, is that prototyping be fast and flexible, and that new architectures and other extensions be easy to explore and test. Although $\mathrm{C}++$ allows the fine control over machine resources that is necessary for peak performance, it is difficult to write, debug and maintain complex systems in $\mathrm{C}++$. 
To provide flexibility, the bulk of the simulator is implemented in the Python scripting language. Python is an interactive high-level language that allows rapid software development and interactive debugging, and includes a wide variety of software libraries for tasks such as data analysis, statistical measurements, and visualization. Unlike the script languages typically included in simulators, Python is a complete, well-defined, mature language with a strong user base. As a result, it enjoys strong support outside of the field of computational neuroscience, which provides much greater flexibility for users as well as making the task of future maintenance easier.

The first full release of Topographica is scheduled for mid-2004, and will include support for Linux, Microsoft Windows, and Macintosh OS X platforms. This release focuses on support for models of vision, but many of the primitives are also usable for auditory and somatosensory models. Included are flexible routines for generating visual inputs (based on geometric patterns, mathematical functions, and photographic images), and general-purpose mechanisms for measuring maps of visual stimulus preference, such as orientation, ocular dominance, motion direction, and spatial frequency maps.

\section{Future research}

Using the tools provided by Topographica, we expect that neuroscientists and computational researchers will be able to answer many of the outstanding research questions about topographic maps, including what roles environmental and intrinsic cues play in map development, and what computations they perform in the adult. Other current research topics include understanding how object segmentation, grouping, and recognition are implemented in maps, and how feedback from higher areas and visual attention affect lower level responses. The simulator is designed throughout to be general and extensible, and so it will also be able to address new research questions that arise from future experimental work.

Several releases of Topographica are planned over the next few years, including user-contributed extensions and models. An online repository will also be set up for user contributions, so that researchers can share code and models. The overall goal is to work towards a common understanding of how topographic maps develop and function.

\section{Conclusion}

The Topographica simulator fills an important gap between existing software for detailed models of individual neurons, and software for abstract models of cognitive processes. The simulator focuses on models formulated at the topographic map level, which is crucial for understanding brain function. We believe this shared, extensible tool will be highly useful for the community of researchers working to understand the large-scale structure and function of the cortex.

\section{Acknowledgments}

Supported in part by the National Institutes of Mental Health under Human Brain Project grant 1R01-MH66991, and by the National Science Foundation under grant IIS-9811478. 


\section{References}

[1] Amari, S.-I. (1980). Topographic organization of nerve fields. Bulletin of Mathematical Biology, 42:339-364.

[2] Bednar, J. A., Kelkar, A., and Miikkulainen, R. (2004). Scaling self-organizing maps to model large cortical networks Neuroinformatics. In press.

[3] Bednar, J. A., and Miikkulainen, R. (2003). Learning innate face preferences. Neural Computation, 15(7):1525-1557.

[4] Bednar, J. A., and Miikkulainen, R. (2003). Self-organization of spatiotemporal receptive fields and laterally connected direction and orientation maps. Neurocomputing, 5254:473-480.

[5] Blasdel, G. G. (1992). Orientation selectivity, preference, and continuity in monkey striate cortex. Journal of Neuroscience, 12:3139-3161.

[6] Bower, J. M., and Beeman, D. (1998). The Book of GENESIS: Exploring Realistic Neural Models with the GEneral NEural SImulation System. Santa Clara, CA: Telos.

[7] Hines, M. L., and Carnevale, N. T. (1997). The NEURON simulation environment. Neural Computation, 9:1179-1209.

[8] Kaas, J. H. (1997). Theories of visual cortex organization in primates. Cerebral Cortex, 12:91-125.

[9] Merzenich, M. M., Knight, P. L., and Roth, G. L. (1975). Representation of cochlea within primary auditory cortex in the cat. Journal of Neurophysiology, 38(2):231-249.

[10] O'Reilly, R. C., and Munakata, Y. (2000). Computational Explorations in Cognitive Neuroscience: Understanding the Mind by Simulating the Brain. Cambridge, MA: MIT Press.

[11] Roque Da Silva Filho, A. C. (1992). Investigation of a Generalized Version of Amari's Continuous Model for Neural Networks. PhD thesis, University of Sussex at Brighton, Brighton, UK.

[12] Swindale, N. V. (1996). The development of topography in the visual cortex: A review of models Network - Computation in Neural Systems, 7:161-247.

[13] Van Essen, D. C., Lewis, J. W., Drury, H. A., Hadjikhani, N., Tootell, R. B., Bakircioglu, M., and Miller, M. I. (2001). Mapping visual cortex in monkeys and humans using surfacebased atlases. Vision Research, 41(10-11):1359-1378.

[14] Weliky, M., Bosking, W. H., and Fitzpatrick, D. (1996). A systematic map of direction preference in primary visual cortex. Nature, 379:725-728. 